\title{
Pulse Combustor Tail-Pipe Heat-Transfer Dependence on Frequency, Amplitude, and Mean Flow Rate
}

\author{
JOHN E. DEC * \\ University of Michigan, \\ Ann Arbor, Michigan
}

and

JAY O. KELLER

Combustion Research Facility, Sandia National Laboratories, Livermore, California

\begin{abstract}
A commonly cited advantage of pulse combustors is a high rate of heat transfer in the tail pipe. Past research on these rates of heat transfer is inconclusive regarding the amount of heat transfer enhancement and how various flow parameters affect this enhancement. This article reports an experimental heat transfer study in the tail pipe of a pulse combustor. The pulsation frequency, pulsation amplitude, and mean flow rate were varied systematically, and their effects on the heat transfer rates assessed. Spatially averaged Nusselt numbers were obtained from thermocouple measurements using a standard log-mean heat exchanger calculation. The Nusselt number was found to increase with both pulsation amplitude and frequency, with a maximum enhancement of 2.5 times that of steady flow at the same mean Reynolds number. The Nusselt number enhancement decreased with increasing mass flow rate for a given combustor pulsation frequency and amplitude. Independent axially resolved heat flux and gas temperature measurements confirmed the large Nusselt number increase with pulsations and demonstrated that entrance effects, although present, were small compared to the Nusselt number enhancement due to the pulsations. The data are compared with quasi-steady theory, which is the only available theory in the literature for this problem. Quasi-steady theory does not account for frequency effects and is not adequate for describing the data from this study.
\end{abstract}

\section{NOMENCLATURE}

\section{A area}

$B \quad$ velocity ratio $V_{\text {osc }} / V_{\text {mean }}$

c average speed of sound in the combustor and tail pipe

$C_{C} \quad$ product of the cooling air mass flow rate and constant pressure specific heat

$d$ derivative

$D \quad$ hydraulic diameter

$h \quad$ heat transfer coefficient (exhaust gas)

$h_{\text {c.a. }} \quad$ heat transfer coefficient (cooling air)

$k \quad$ conductivity of the exhaust gas

* Currently at Sandia National Laboratories, Albuquerque, New Mexico. $k_{\text {wall }} \quad$ conductivity of the wall of the inner tube

$L \quad$ length of the tail pipe

$\mathrm{Nu} \quad$ Nusselt number (exhaust gas), $h D / k$

$P_{\max } \quad$ combustion chamber pressure oscillation amplitude

$\boldsymbol{P}_{\mathrm{RMS}} \quad$ combustion chamber pressure root mean square about the mean

Pr Prandtl number (exhaust gas)

$Q \quad$ heat flux from exhaust gas to cooling air

$Q_{0} \quad$ heat flux from cooling air to ambient

$\overline{\operatorname{Re}}$ mean Reynolds number of the exhaust gas, based $V_{\text {mean }}$ and $D$

$t_{\text {wall }} \quad$ wall thickness of the inner tube of the tail pipe

$\Delta T_{1} \quad$ temperature difference between the exhaust gas and cooling air at the combustion chamber end of the tail pipe 
$\Delta T_{2}$ temperature difference between the exhaust gas and cooling air at the decoupling chamber end of the tail pipe

$U$ overall heat transfer coefficient, $1 / U=$ $1 / h+t / k_{\text {wall }}+1 / h_{\text {c.a. }}$.

$V_{\text {mean }}$ mean tail-pipe velocity, cycle-averaged velocity

$V_{\text {osc }} \quad$ tail-pipe velocity oscillation amplitude

$V_{\text {osc-avg }}$ spatially averaged tail-pipe velocity oscillation amplitude

$V_{\text {osc-max }}$ maximum tail-pipe velocity oscillation amplitude, occurs at the decoupling chamber end

$x \quad$ spatial variable, along the axis of the tail pipe, $x=0$ is the contraction section, tail pipe interface.

\section{Greek Symbols}

$\lambda$ wavelength of the fundamental frequency of the combustor at the average combustor and tail pipe gas temperature $\omega \quad$ frequency variable

$\omega_{0} \quad$ minimum frequency value for frequency enhancement effects

$\rho$ average density of the gases in the combustor and tail pipe

$\tau \quad$ normalized time variable, real time normalized by the period of a cycle

\section{INTRODUCTION}

The need for highly efficient combustion systems has led to active research in pulse combustion. Pulse combustion heating systems have many advantages over conventional burners, such as thermal efficiencies of $95 \%$ or more, low pollutant $\left(\mathrm{NO}_{x}\right.$ and $\mathrm{CO}$ ) emissions, and self aspiration. A high rate of convective heat transfer in the tail pipe is another commonly cited advantage of pulse combustors. Some previous research indicates that these heat transfer rates may be significantly greater than those of steady turbulent flow at the same mean Reynolds number. However, due to a lack of understanding of the heat transfer and other fundamental processes, the design of pulse combustors has proceeded largely by trial and error.
As a result, there has been little design optimization, and these efficient burners have not been adapted for use in many potential markets, particularly, large-scale commercial applications. More complete discussions of pulse combustion applications and research needs may be found in recent review articles [1, 2]. An understanding of the heat transfer in the tail pipe is important in the design of pulse combustors for two reasons. First, the heat transfer affects the fundamental pulsation mechanism of the combustor through changes in the gas temperature, and, second, an understanding of the heat transfer would permit optimization of the heat transfer enhancement, thus leading to more efficient, compact heat exchangers on pulse combustor heating systems.

The large flow oscillations in the tail pipe are generally believed to be responsible for the observed heat transfer effects. (These flow oscillations result from the acoustic resonance of the combustor, and no external pump work is required.) The results of previous research have been inconclusive as to the effect of flow oscillations on heat transfer. Convective heat transfer coefficients in pulse combustor tail pipes have been found to vary from $70 \%$ less [3] to $240 \%$ greater [4] than those for steady flow conditions. Research on other oscillating flows has shown decreases in the heat transfer coefficient of up to $20 \%$ [5] and increases of up to a factor of 5 [6] over steady flow conditions. The degree of enhancement reported in the literature is sometimes confusing because in some studies the pulsating heat transfer is compared to steady flow conditions in the same device, which are not always fully turbulent, while in others it is compared with heat transfer rates predicted from turbulent flow correlations. These inconsistencies undoubtedly account for some of the apparent discrepancies; however, more significant is that the flow parameters differed greatly between these studies. In addition, many studies in the literature have been at frequencies much lower than the $45-200-\mathrm{Hz}$ range, typical of pulse combustors. In none of the previous studies at pulse combustor frequencies (except for a presentation of the current work [7]) were all the important flow parameters, oscillation frequency, velocity oscillation amplitude, and 
mean flow rate varied systematically, so that their effects on the heat transfer could be determined.

This article reports the result of an experimental study of the heat transfer in a pulse combustor tail pipe, in which pulsation frequency, pulsation amplitude, and mean flow rate were systematically varied. Systematic control of the basic flow parameters is unique in pulse combustor studies, and was possible because of recent developments in understanding the characteristic times of the pulse combustor, reported by Keller et al. [8, 9]. This new understanding allowed the test combustor to be operated from 54 to $101 \mathrm{~Hz}$ with pulsation amplitudes of the combustion chamber pressure varying from near zero (steady flow) to $23 \mathrm{kPa}$ peak-to-peak (peak-to-peak velocity oscillations of 10 times the mean) and mean flow Reynolds numbers from 3100 to 4750 . Although all three flow parameters could not be varied with complete independence, experiments were conducted over a sufficient range to determine the trends resulting from each parameter. The heat transfer effect at each operating condition is determined from a spatially averaged Nusselt number obtained using standard heat exchanger data reduction techniques, as will be discussed later. In addition, axially resolved heat flux and Nusselt numbers were obtained at some conditions. These axially resolved measurements allowed an assessment of the magnitude of entrance effects on the Nusselt number, and provided an independent check on the spatially averaged measurements.

\section{EXPERIMENTAL FACILITY AND DIAGNOSTICS}

\section{Experimental Facility}

The pulse combustor used in this study was a "Helmholtz" type with an external flapper valve. A schematic of this combustor, with typical dimensions, is shown in Fig. 1. This combustor consists of an axisymmetric injection or "mixing" chamber, and square cross-section combustion chamber, contraction section, and tail pipe. Acoustic termination for the tail pipe was provided by a large-volume $\left(0.031 \mathrm{~m}^{3}\right)$ decoupling chamber. Reactants were premixed upstream of the flapper valve and injected into the "mixing" chamber by one of two methods, either tangentially, which created a large swirl component in the tail pipe flow, or on axis, as shown in the figure. Time-averaged reactant mass flow rates were metered and controlled with sonic nozzles. The reactants consisted of pure methane and air at an equivalence ratio of 1.0 , and a variable fraction of nitrogen diluent, which was used to control the energy release rate as discussed below.

The tail pipe consisted of a coaxial square pyrex tube arrangement, as shown in the figure. In this arrangement, the inner tube, which was $30 \mathrm{~mm}$ square with a wall thickness of $4.75 \mathrm{~mm}$, formed the resonance tube for the pulse combustor and carried the combustion products. The outer tube was $51 \mathrm{~mm}$ square, which provided a $5.75-\mathrm{mm}-$ wide channel between the tubes on all four sides. Cooling air flowed through this square annular space between the inner and outer tubes, entering at the decoupler end of the tail pipe and exiting at the contraction section end. This tube arrangement formed a counterflowing heat exchanger. The magnitude of the cooling-air heat-transfer coefficient was double that of the highest exhaust-gas heat-transfer coefficient measured in this study, insuring sensitivity to desired measurement. The tail pipe was constructed in a modular fashion, allowing its length to be varied in $200-\mathrm{mm}$ increments from 680 to $1680 \mathrm{~mm}$, to obtain operating frequencies.from 101 to $54 \mathrm{~Hz}$ respectively. Modular construction also permitted the insertion of special test sections, described below, at $200 \mathrm{~mm}$ increments. Axial positions, given by the $x$ coordinate, are measured from the tail pipe entrance towards the exit as shown in Fig. 1.

\section{Pulse Combustor Control}

This study required the pulse combustor to be operated over a range of frequencies and pulsation amplitudes. Combustor frequencies were varied by changing the natural resonance frequency through variations in the tail pipe length, and subsequent adjustment of the timing of the energy release rate. The timing of the energy release rate for this premixed system is controlled by the mixing rate of the reactants with the hot products 


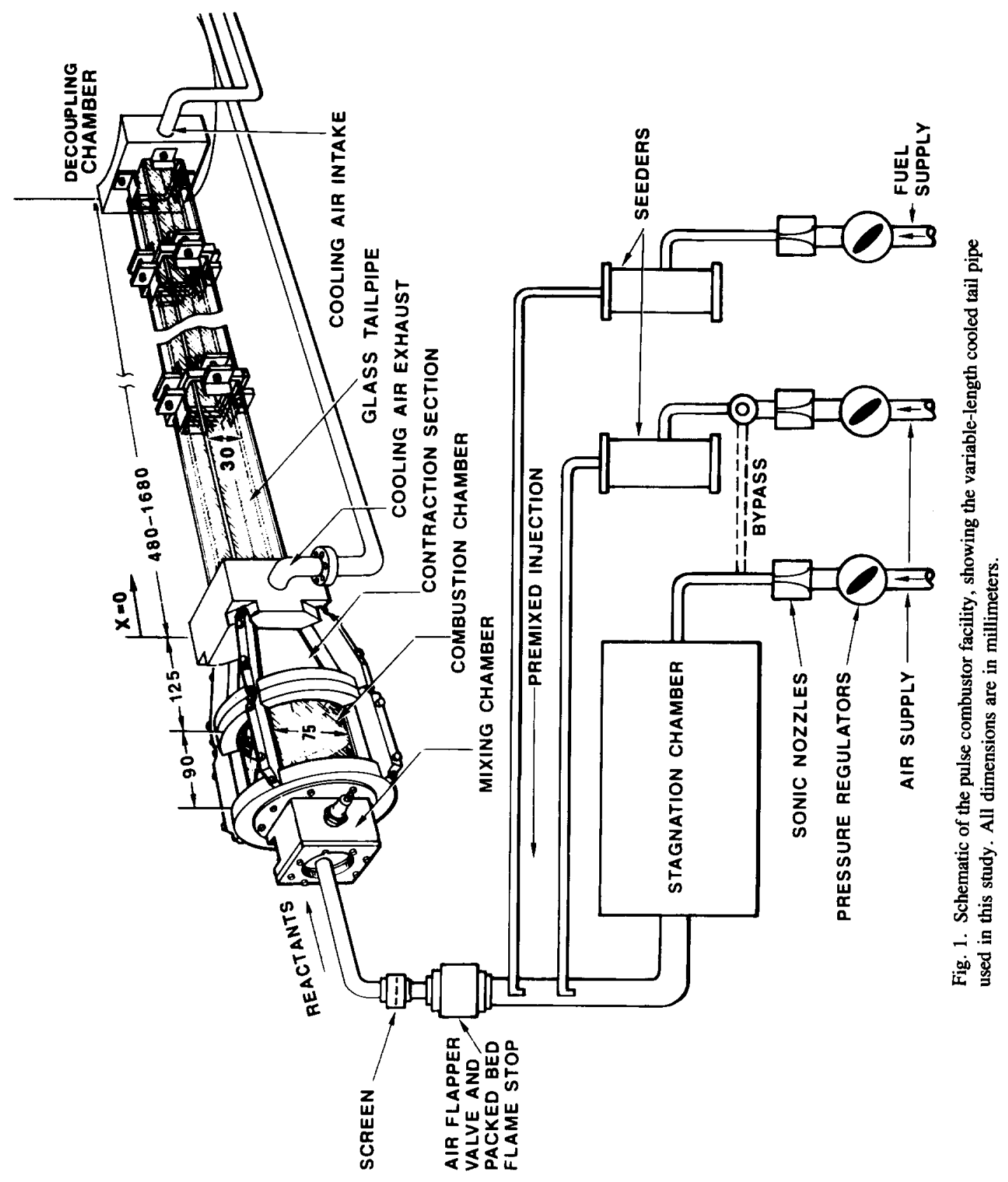


and the chemical reaction rate. By adjusting these two processes, the energy release was optimally timed for each natural resonance frequency, providing a maximum pulsation amplitude. Pulsation amplitudes were then decreased from this maximum by detuning the timing of the energy release through the addition of various amounts of nitrogen diluent to the reactants. Adding nitrogen slowed down the chemical reaction rate, which delayed the energy release rate. By this method, a range of pulsation amplitudes were obtained for each frequency. The effect was sufficiently strong to stop the pulsations completely and achieve steady flow. A complete discussion of this methodology for controlling the pulse combustor may be found in Keller et al. [8, 9], and Bramlette [10] discusses jet mixing concepts in the pulse combustor.

\section{Diagnostics}

Pressure transducers, laser Doppler velocimetry (LDV), and thermocouples were the diagnostics used in this study. Pressure transducers were used to determine the combustor frequency and to measure the combustion chamber pressure. The root mean square about the mean of the combustion chamber pressure $\left(P_{\mathrm{RMS}}\right)$ was used as a measure of pulsation amplitude. LDV was used to measure a typical oscillating gas velocity in the tail pipe; a complete discussion of the technique may be found in Dec and Keller [11]. Thermocouples were installed to measure the tail pipe entrance and exit temperatures of the exhaust gas and cooling air. All temperatures reported are time averaged over many combustor cycles. Fine wire $(50 \mu \mathrm{m})$ thermocouples were used in the exhaust gas and a radiation correction applied as described below. The thermocouples used at the exhaust gas inlet and exit were made of $\mathrm{Pt}-\mathrm{Pt}, 13 \% \mathrm{Rh}$ and chromel-alumel, respectively. Because the cooling air was never greater than $250^{\circ} \mathrm{C}$, radiation corrections were not required and sheathed chromel-alumel thermocouples were used.

Radiation corrections were applied to the exhaust gas thermocouple measurements, using a simple energy balance. A spherical heat transfer correlation was used to calculate the convective heat transfer coefficient. To account for the flow oscillations, the Reynolds number used in this correlation was taken to be twice that of the timeaveraged value for the tail pipe. As the Nusselt number for very small diameter spheres is a weak function of the Reynolds number, errors from this assumption are small. An emissivity of 0.2 was used for the uncoated Pt-Pt, 13\% Rh thermocouple, as suggested by Bradley and Entwistle [12]. The transmissivity of quartz to thermal radiation from a gray body at the temperature of the exhaust gas was obtained from a transmissivity curve. Errors in the corrected gas temperature due to assumptions required for the radiation correction were small, since the maximum correction was $2.5 \%$ of the gas temperature.

Axially resolved measurements were made using two movable test sections, which were 200 $\mathrm{mm}$ long. One test section had an access port that allowed a thermocouple to be inserted into the exhaust gas at the axial center of the test section. Radiation corrections were also applied to this thermocouple, which was identical to the thermocouple used at the exhaust gas inlet. The second test section was equipped with a thin-film thermocouple, vacuum deposited on the exhaust gas side surface of the inner tube, to measure wall temperature and wall heat flux. This thermocouple, made of pure platinum-pure rhodium, was extremely thin (approx. $10 \mathrm{~nm}$ ), so that its effect on the heat flux and wall temperature was negligible. The junction of the thin film thermocouple was approximately $2 \mathrm{~mm}$ square, and was located at the axial and transverse center of one wall of the test section. A second thermocouple was imbedded in the wall directly opposite the junction of the thinfilm thermocouple. The thickness of the wall between the two thermocouples was measured, allowing the heat flux to be calculated. The inner tubes of these two test sections were made of fused silica quartz rather than pyrex. The $17 \%$ difference in the thermal conductivities of these two materials produced only a negligible $2.5 \%$ difference in the overall heat transfer coefficient, which is dominated by the convective terms.

\section{THEORETICAL CONSIDERATIONS}

Pulse combustors are devices in which the combustion process drives an acoustic resonance. For the combustor used in this study the acoustic 

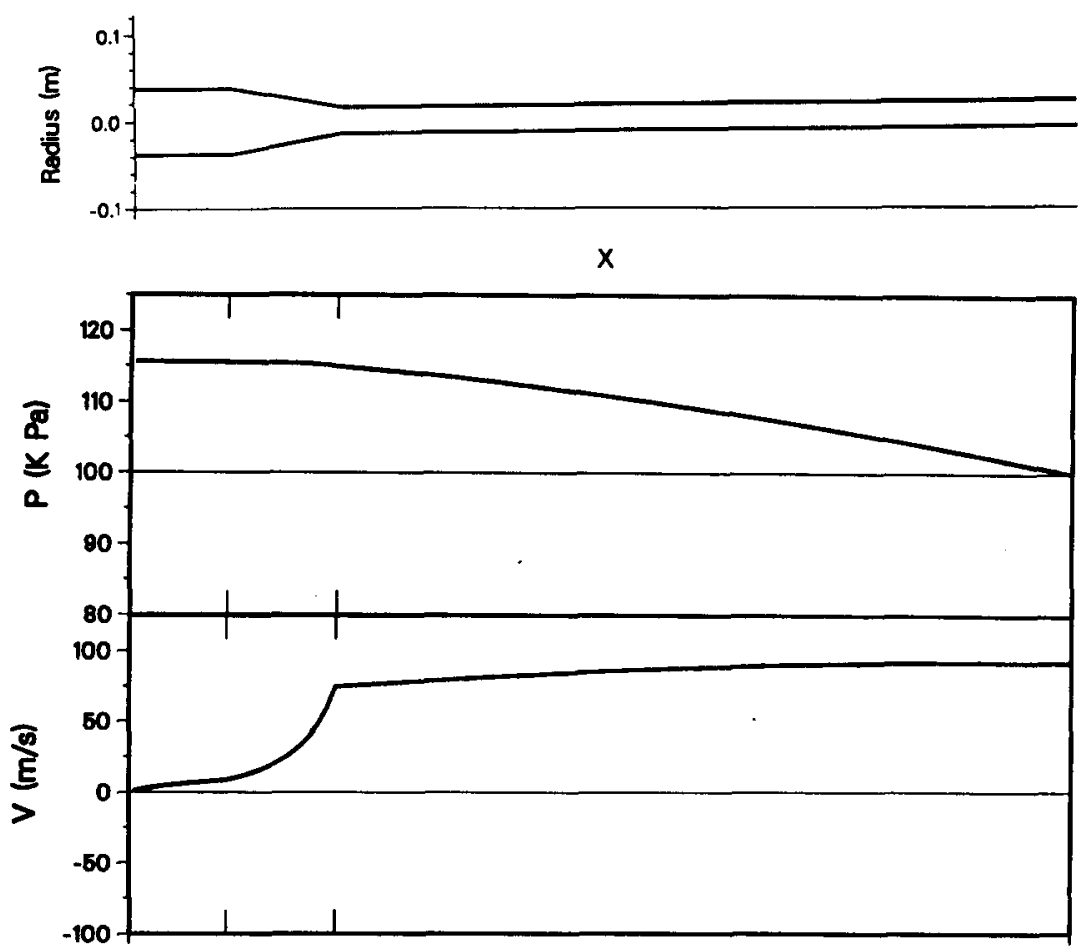

Fig. 2. Outline drawing of the pulse combustor, and typical plots of the oscillating pressure and velocity distribution at times of maximum oscillation.

resonance is roughly that of a quarter-wave tube with variable area. An outline drawing of this pulse combustor and plots of the maximum oscillating pressure and velocity waves generated by the acoustic resonance are shown in Fig. 2. At the combustion chamber end are a pressure antinode and velocity node, while at the tail pipe exit are a pressure node (constant at atmospheric pressure) and velocity antinode. The acoustic resonance creates velocity oscillations superimposed on the mean flow in the tail pipe. These velocity oscillations can be large, with amplitudes up to 5 times the mean velocity (see Fig. 3). The amplitude of these velocity oscillations is greatest at the tail pipe exit and decreases sinusoidally toward the tail pipe entrance. Because of the area change between the combustion chamber and tail pipe, the velocity oscillations are still significant at the tail pipe entrance (see Fig. 2), with acoustic theory predicting the oscillation amplitude at the entrance to be $83 \%$ of that at the exit.

The combustion chamber pressure and tail-pipe velocity oscillations, ensemble averaged over a

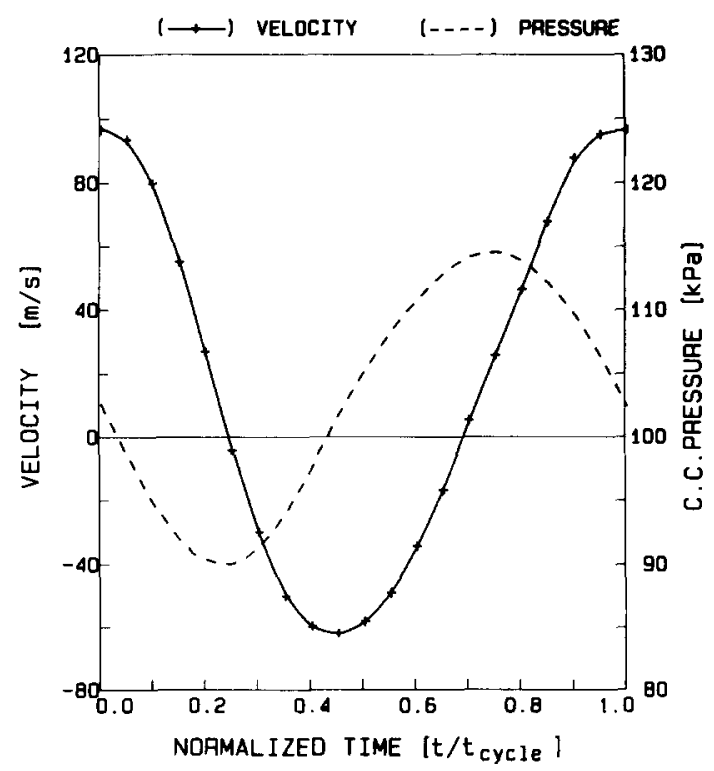

Fig. 3. Streamwise velocity and combustion chamber pressure during a combustor cycle at a combustor frequency of $83 \mathrm{~Hz}$. These data are ensemble-averages of a minimum of 400 combustor cycles. The velocity measurements were taken at the tail pipe centerline $540 \mathrm{~mm}$ from the tail pipe entrance. 
minimum of $\mathbf{4 0 0}$ combustor cycles are plotted in Fig. 3. These values are plotted against normalized time (time normalized by the period of a complete cycle). Both waveforms are sinusoidal and are out-of-phase by approximately one quarter of a cycle. This is in agreement with acoustic theory for quarter-wave-tube resonance. Acoustic wave equations show that the amplitude of the velocity oscillations in the tail pipe is directly related to the amplitude of the combustion chamber pressure oscillations. This prediction has been experimentally verified in the pulse combustor by Dec and Keller [11]. In the present article the term pulsation amplitude refers to both pressure and velocity oscillation amplitude.

Superimposed on the large velocity oscillations

- created by the acoustic resonance, the flow in the tail pipe is expected to have small random or "turbulent" fluctuations. Mean flow Reynolds numbers are sufficiently high, $3100-4750$, that laminar flow would not be expected even with no flow oscillations. Moreover, the velocity oscillations periodically create Reynolds numbers that are up to 5 times these mean values. Although the effect of flow oscillations on the nature of the random velocity fluctuations (turbulence) is not known, the large velocity shears associated with these flow oscillations would be expected to produce additional turbulence. Since this flow is turbulent as well as oscillating, the heat transfer results are compared with those of steady (not oscillating) turbulent flow.

\section{Quasi-Steady Theory}

Most attempts to solve the heat transfer analytically or numerically resort to using steady or quasi-steady assumptions. (See, for example, Refs. 13 and 14.) Quasi-steady, as applied to oscillating flows, means that at any point in the cycle the flow is assumed to behave as if it were steady at the instantaneous velocity. This assumption can only be valid for flows with low frequencies or small oscillation amplitudes, since it requires that the flow come to fully developed equilibrium within a time much less than the cycle time.

Although the frequency and amplitude range of the oscillations in the pulse combustor tail pipe are beyond the quasi-steady limit, it is the only theory available, and therefore widely used in the literature. Quasi-steady concepts have been applied to oscillating flow heat transfer in different ways, such as through the adaption of $\mathbf{k}-\epsilon$ turbulence models [14]. However, the most common approach in pulse combustor heat transfer is to apply quasi-steady concepts directly to a heat transfer correlation by substituting in the absolute value of the instantaneous velocity and integrating over a cycle. The first known use of this approach was by Martinelli et al. in 1943 [15]; since then it has been used by several other researchers $[4,5,16]$. Equation 1 shows the quasi-steady adaptation of the Colburn correlation for turbulent heat transfer.

$$
\begin{aligned}
\mathrm{Nu}= & 0.023 \operatorname{Pr}^{1 / 3} \overline{\operatorname{Re}}^{0.8} \\
& \times \int_{0}^{1}(|1+B \cos (2 \pi \tau)|)^{0.8} d \tau .
\end{aligned}
$$

Here $\mathrm{Nu}, \mathrm{Pr}$, and $\overline{\mathrm{Re}}$, are the Nusselt number, Prandtl number, and Reynolds number based on the mean velocity, respectively, and the oscillating velocity is assumed to have a sinusoidal shape (an assumption verified by the velocity measurements in Fig. 3 and Ref. 11). $B$ is the ratio of the velocity oscillation amplitude to the mean velocity ( $V_{\text {osc }}$ ) $V_{\text {mean }}$ ) and $\tau$ is the time normalized by the time for a complete cycle. Since all heat transfer data to date have been averaged over many cycles, only cycle-integrated results of Eq. 1 have been compared with experiments. Used in this manner, quasi-steady theory has been found, in some cases, to provide reasonably good agreement with experiment well beyond the theoretical quasi-steady limits [4].

Two additional assumptions are implicit in using the quasi-steady Nusselt number correlation shown in Eq. 1. First, steady-flow heat transfer correlations, such as the Colburn correlation used above, are based on Reynolds analogy between momentum and energy. The validity of this analogy in the reversing, oscillating flow of a pulse combustor tail pipe is unknown. Second, Nusselt number correlations developed for steady pipe flow, such as the Colburn correlation, assume 
that the pressure gradient is favorable, i.e., pressure decreases in the direction of the flow. This is not the case for a flow in which the velocity oscillations are driven by the pressure, such as the acoustically resonant flow in the pulse combustor tail pipe. From the combustion chamber pressure and tail pipe velocity profiles shown in Fig. 3 it can be seen that the pressure gradient is adverse for half the cycle. The assumptions of quasi-steady behavior and Reynolds analogy in flows that strongly oscillate at pulse combustor frequencies are questionable, and the assumption of a continuously favorable pressure gradient is clearly invalid. The magnitude of the effects of these assumptions on the Nusselt number is unknown.

Despite these shortcomings of quasi-steady theory, it is the only theory available in the literature for oscillating flow heat transfer, and the experimental results from this study are also compared with the quasi-steady heat transfer correlation presented in Eq. 1. The value of $V_{\text {osc }}$ was taken to be the average velocity oscillation amplitude in the tail pipe, determined from $\boldsymbol{P}_{\mathrm{RMS}}$ using linear acoustic theory, as shown in Appendix A. At a typical operating condition, this value of $V_{\text {osc }}$ was found to be $19 \%$ less than the value obtained directly from velocity measurements. The value calculated from the combustion chamber pressure is used, since it is more comparable with the quasisteady theory presented in most previous works, which obtained $V_{\text {osc }}$ by similar means [4]. In the current article the convective heat transfer rate is given in terms of the Nusselt number defined as $h D / k$, where $h$ is the heat transfer coefficient, $D$ the hydraulic diameter of the pipe, and $k$ the conductivity of the gas.

Fluid properties were taken to be those of mixtures of $\mathrm{CO}_{2}, \mathrm{H}_{2} \mathrm{O}$, and $\mathrm{N}_{2}$ resulting from complete combustion of the reactants, with the temperature evaluated as follows. For the logmean data reduction and quasi-steady theory predictions, a spatially averaged film temperature based on the inlet and exit temperatures of the exhaust gas and cooling air was used. For the axially resolved data, an axially resolved film temperature was computed from the axially resolved gas temperature, and the wall temperature obtained with the thin-film thermocouple. The speed of sound and gas density required for the acoustic relations between pressure and velocity oscillations, used in the comparisons of quasisteady theory with the data, were evaluated at the spatially averaged gas temperature. This assumption of constant gas properties in the acoustic relations undoubtedly contributes to the discrepancy between the calculated and measured values of $V_{\text {osc }}$ noted above.

\section{Nusselt Number Calculations}

Axially resolved Nusselt numbers were calculated for some conditions. Using the two wall-thermocouple measurements, the local heat flux was calculated by solving the conduction equation in the wall. Combining this result with the local gas temperature and wall surface temperature measurements, the exhaust gas Nusselt number was calculated directly. The energy balance between the sum of these local heat fluxes times their respective wall areas, and the total enthalpy difference of the exhaust gas from tail pipe inlet to exit was checked for pulsing and steady flows at a typical operating condition. For the pulsing flow the energy balance closed within $2.4 \%$. For the steady flow the sum of the axially resolved measurements overpredicted the total energy loss by $16.6 \%$. This larger error of closure is believed to be due to a lower heat flux in the corners of the tail pipe under steady flow conditions, which are likely not fully turbulent, as is discussed later. The contribution of gas radiation to the heat transfer was calculated to be a maximum of $10 \%$ and was neglected.

Spatially averaged Nusselt numbers were calculated from the exhaust gas and cooling air inlet and exit temperatures using a log-mean formula for a counterflowing heat exchanger. Typically, the derivation of this formula assumes that the heat exchanger is insulated [17]. Because the outer wall of the tail pipe was not insulated, the log-mean formula was derived accounting for heat loss to the ambient. The result of this nonadiabatic derivation may be reduced to the adiabatic expression for the overall heat transfer coefficient, $U$, as discussed in the Appendix B. To determine the desired exhaust gas Nusselt number, the exhaust gas heat transfer 
coefficient must be obtained from $U$ by removing the contributions from the convection coefficient for the cooling air and wall conduction, as

$\frac{1}{h}=\frac{1}{U}-\frac{1}{h_{\text {c.a. }}}-\frac{t_{\text {wall }}}{k_{\text {wall }}}$,

where $h$ is the desired exhaust gas heat transfer coefficient, $h_{\text {c.a. }}$ is the cooling air heat transfer coefficient, and $k_{\text {wall }}$ and $t_{\text {wall }}$ are the conductivity and thickness of the wall, respectively. The wall conductivity was evaluated at the mean wall temperature, and the cooling air heat transfer coefficient was determined from the axially resolved data. This coefficient was 2.4 times greater than that predicted by a turbulent pipe-flow heattransfer correlation that accounted for entrance effects, using a cooling air Reynolds number, based on the hydraulic diameter, of 5250. A check on this high value of the coefficient, obtained by varying the cooling air flow rate, showed the factor of 2.4 (times the value from the correlation) to give consistent results.

\section{RESULTS AND DISCUSSION}

\section{Axially Resolved Study}

Axially resolved gas temperatures, wall heat flux, and Nusselt numbers for steady and pulsing flow are shown in Figs. 4 and 5, respectively. These data were taken using axial injection for the 880 $\mathrm{mm}$ tail pipe. The pulsing case had a frequency of $83 \mathrm{~Hz}$ with a $P_{\mathrm{RMS}}$ of $7.35 \mathrm{kPa}$. Although the mean mass flow rate was the same for the steady and pulsing cases, the mean Reynolds numbers were 3500 and 3750 respectively, due to temperature variations in the dynamic viscosity. The data points shown are the mean values from several different data sets, and the error bars indicate the maximum and minimum values obtained. Examining the steady flow data in Fig. 4, the Nusselt number is roughly constant at about 15 , which is close to the value of 14.4 predicted for steady turbulent flow by the Colburn correlation. The pulsing data in Fig. 5 is distinguished from that of the steady flow by two main features. First the drop in gas temperature is greater with a corres-

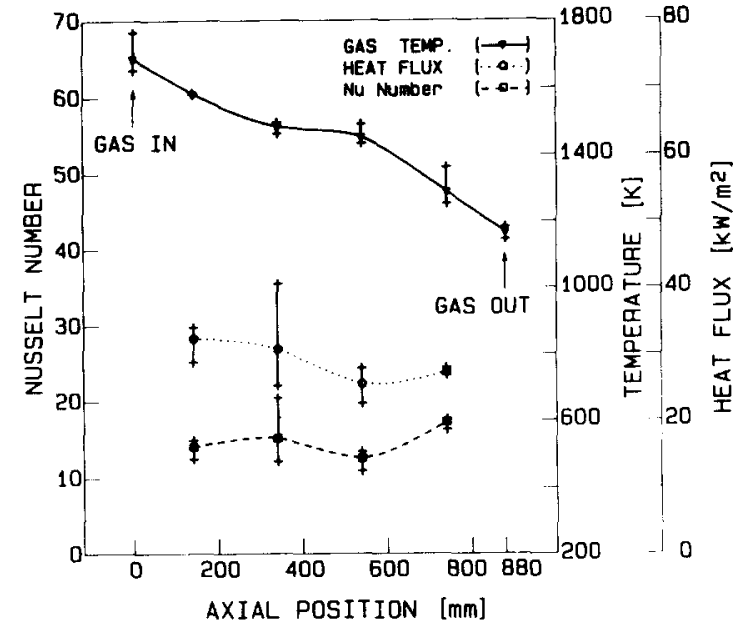

Fig. 4. Nonpulsing-flow Nusselt number, gas temperature, and heat flux distribution along the tail pipe. Tail pipe length is $880 \mathrm{~mm}$. The heat flux scale is different from that in Fig. 5 . Data points are the mean values of several data sets, and the error bars indicate the maximum and minimum values obtained. The lines are cubic spline fits to the points shown.

ponding increase in heat flux (note the heat-flux scale change in these two plots). Second, the Nusselt number is a factor of 2 greater. The Nusselt number remains fairly constant at about 30 (also double the value of 15.3 predicted by the

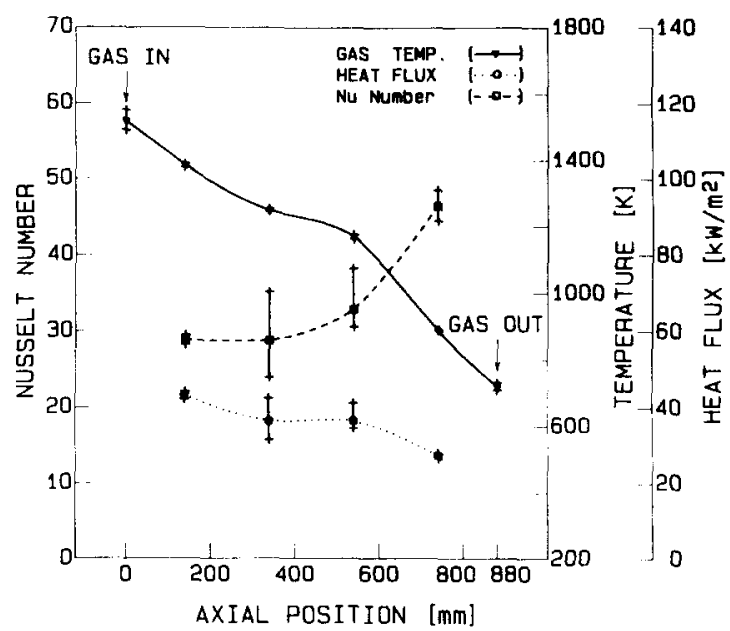

Fig. 5. Pulsing-flow Nusselt number, gas temperature, and heat flux distribution along the tail pipe. Tail pipe length is $\mathbf{8 8 0}$ $\mathrm{mm}$, frequency is $83 \mathrm{~Hz}$, and combustion chamber pressure RMS is $7.35 \mathrm{kPa}$. The heat flux scale is different from that in Fig. 4. Data points are the mean values of several data sets, and the error bars indicate the maximum and minimum values obtained. The lines are cubic spline fits to the points shown. 
Colburn correlation) for the first three test-section locations ( $x=140,340$, and $540 \mathrm{~mm})$, with a large increase at the last test-section location ( $x=$ $740 \mathrm{~mm}$ ). This higher Nusselt number at the exit is attributed to entrance effects during flow reversal. As seen in Fig. 3, periodic flow reversals are large and extend over a significant fraction of the cycle. The tail pipe exit has an abrupt transition into the decoupling chamber; thus during flow reversal it acts as a square-edged entrance, producing a higher Nusselt number. Entrance effects were not seen at the inlet, most likely because the contraction section provides a smooth transition from the combustion chamber to the tail pipe. Reay [18] made axially resolved heat-transfer measurements along the tail pipe of a Schmidt tube pulse combustor and noted similar increases at both the inlet and exit region. This is in agreement with the current findings, since the flow near the exit of a Schmidt tube combustor is similar to that of the "Helmholtz" type combustor used here.

The entrance effect at the tail pipe exit, although locally significant, adds only slightly to the overall Nusselt number enhancement caused by the pulsations. The Nusselt number measured at the center of exit section $(x=740 \mathrm{~mm})$ is approximately 45 , which is $50 \%$ greater than the value of 30 along the rest of the tail pipe. However, since this entrance effect appears to penetrate in only 200$300 \mathrm{~mm}$ (see Fig. 5) from the tail-pipe exit, the spatially averaged Nusselt number is only $17 \%$ higher than the value of 30 found at the first three test sections locations. This $17 \%$ average increase is of similar magnitude to the $25-30 \%$ increase predicted by correlations for steady turbulent pipe flow for the length-to-diameter ratios used in this study. Thus, although these entrance effects add to the observed increase in Nusselt number for the pulsing case, their effect in this tail pipe is small compared to the factor of 2 increase over the expected value of 15.3 for steady turbulent flow. Furthermore, entrance effects alone are not sufficient to explain the large Nusselt number increases seen in this type of oscillating flow. The entrance effect will also cause some discrepancies in the variable frequency data, presented below, because the different combustor frequencies were obtained by varying the tail pipe length, with a constant combustion chamber volume. This variation was calculated to be less than $5 \%$ between the 1680 and $680-\mathrm{mm}$ tail pipe lengths, which is small compared to the observed Nusselt number variation (see discussion below), and was neglected.

\section{Pulsation Amplitude and Frequency Effects}

Time- and space-averaged Nusselt numbers, calculated by the log-mean heat exchanger correlation described above, are presented in Figs. 6 and 7. Figures 6 and 7 contain data obtained using swirl and axial injection, respectively. The axial injection is preferred for this study, since it created a uniform, nearly one-dimensional flow, while the swirl injection resulted in a strong swirl in the tail pipe flow. Swirl data are included for the lower frequencies, since the pulse combustor would not operate stably at frequencies below $67 \mathrm{~Hz}$ with axial injection. In these figures, Nusselt numbers for various combustor frequencies are plotted against pulsation amplitude as measured by $\boldsymbol{P}_{\mathrm{RMS}}$. Each line corresponds to a different tail pipe length and, hence, combustor frequency, as given on the

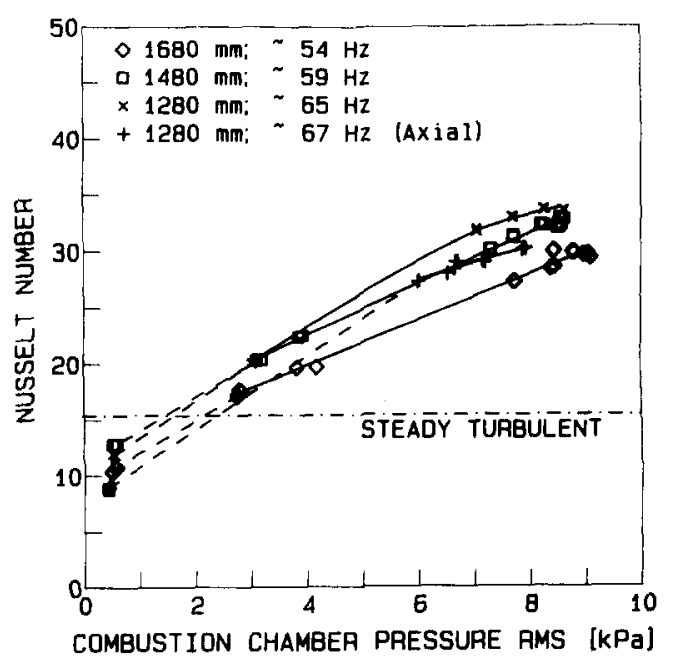

Fig. 6. Swirl injection; time- and space-averaged Nusselt numbers for various frequencies as a function of combustion chamber pressure RMS. Also shown is the Nusselt number for steady turbulent flow at the same mean Reynolds number. Dashed lines indicate regions where data are insufficient to accurately predict Nusselt number variation with pulsation amplitude. The $67 \mathrm{~Hz}$ data was taken with axial injection, and is shown for comparison. 


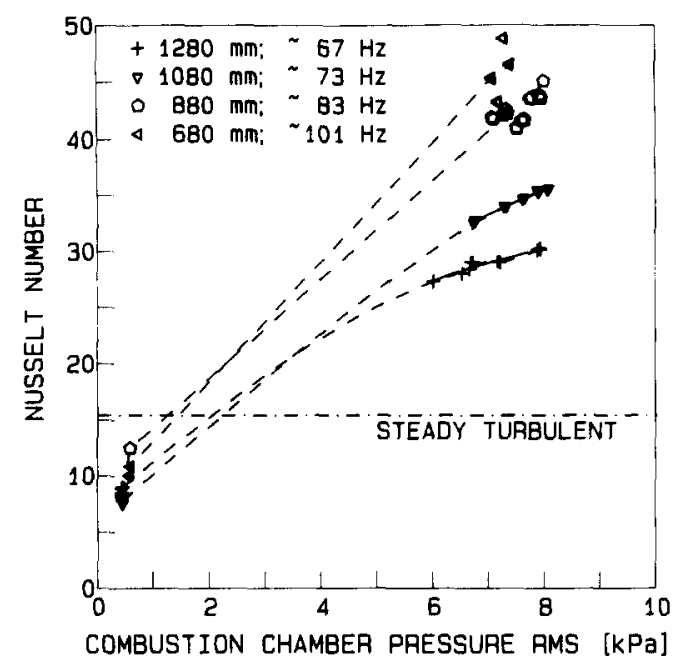

* Fig. 7. Axial injection; time- and space-averaged Nusselt numbers for various frequencies as a function of combustion chamber pressure RMS. Also shown is the Nusselt number for steady turbulent flow at the same mean Reynolds number. Dashed lines indicate regions where data are insufficient to accurately predict Nusselt number variation with pulsation amplitude.

figure. The data plotted at the lowest $P_{\mathrm{RMS}}$ are for the nonpulsing or steady-flow case. All of these data are at the same mean mass flow rate; however, temperature effects on the dynamic viscosity resulted in some variation in mean Reynolds number. For the pulsing cases the mean Reynolds number varied from 3650 to 4050 , with an average value of 3850 , and for the nonpulsing cases it varied from 3500 to 3600 , with an average value of 3550 . Also shown in the figures is the value of the Nusselt number predicted from the Colburn correlation for steady turbulent flow at the mean Reynolds number of the pulsing cases, 3850.

All the Nusselt numbers for the nonpulsing cases fall below the value of 14.5 predicted by the Colburn correlation for a Reynolds number of 3550 and above the laminar value of 3.6 for square ducts with constant heat flux at the wall [17]. This result is reasonable since flows of this Reynolds number, in smooth pipes, fall in the transition regime between laminar and fully turbulent flows. Also, the log-mean Nusselt numbers are lower than the value of 15 seen in the axially resolved data (Fig. 4). Laminar flows, and to a much lesser extent turbulent flows, in rectangular ducts are known to have convective coefficients that vary around the periphery, approaching zero in the corners [17]. Because this flow appears to be in the transition regime, it is reasonable that the logmean Nusselt numbers, which are averages over the entire wall area, are smaller than the axially resolved values, which were measured in the center of a wall. This effect of the square duct also explains the $16.6 \%$ closure error in the energy balance noted in the previous section. Finally, all of the nonpulsing cases do not have the same value of the Nusselt number. This is a result of a very small residual resonance for some cases. In this $P_{\mathrm{RMS}}$ range, the Nusselt number increases rapidly with very small increases in $P_{\mathrm{RMS}}$, probably due to a transition towards fully turbulent flow.

In contrast to the nonpulsing cases, the Nusselt number for virtually all the pulsing cases is significantly higher than the predicted steady flow value. At all frequencies the Nusselt number increases roughly linearly with increases in $P_{\mathrm{RMS}}$, which corresponds to increases in the velocity oscillation amplitude. This increase is significant for all frequencies shown, but greatest at the highest frequencies. Also, the rate of increase of the Nusselt number with pulsation amplitude does not decrease significantly at the maximum pulsation amplitudes. For the swirl injection cases (Fig. 6), which are for frequencies ranging from 54 to $65 \mathrm{~Hz}$, the maximum enhancement is about 2 times that predicted for steady turbulent, nonswirling flow. In order to quantify the heat transfer effects due to the swirl alone, the lowest frequency obtainable with axial injection, $67 \mathrm{~Hz}$, is also presented in Fig. 6 for reference. This axial injection case, which lacks the convection resulting from the swirl, has a slightly lower Nusselt number than the corresponding $65-\mathrm{Hz}$ swirl case. Figure 7 presents the axial injection data, which has a maximum Nusselt number enhancement, at $101 \mathrm{~Hz}$, nearly triple the predicted steady-flow value. The range of pulsation amplitudes at which the combustor would operate stably was limited with the axial injector, resulting in a dearth of data at low to intermediate pulsation amplitudes, especially at the higher frequencies. The lines connecting these data points are dashed to indicate that 
insufficient data exist to accurately predict the Nusselt number behavior at these pulsation amplitudes. The crossing of some of these dashed lines does not indicate real trends, but results from a lack of sufficient data and the variation in the Nusselt numbers for the nonpulsing cases as previously discussed.

A comparison of the axially resolved Nusselt numbers in Fig. 5, and the log-mean Nusselt number for the same operating condition in Fig. 7 shows a discrepancy of $20 \%$. The axially resolved data yields an axially weighted-average Nusselt number of $\mathbf{3 4 . 7}$ for this operating condition, while the log-mean calculation gives a Nusselt number of 42.6 for this same condition. The log-mean Nusselt number formula assumes that the Nusselt number is constant along the tail pipe, an assumption that Fig. 5 shows is not valid near the end of the tail pipe. Comparisons of the log-mean Nusselt numbers with directly measured, axially resolved Nusselt numbers at several other frequencies and pulsation amplitudes showed similar systematic discrepancies. Therefore, all the trends shown by the log-mean Nusselt numbers may be considered correct, but the values are about $20 \%$ high, resulting in a true maximum enhancement factor of 2.5 .

The effect of frequency on the heat transfer enhancement may be seen more clearly in Fig. 8, which shows Nusselt numbers for several frequencies at the same $P_{\mathrm{RMS}}$, approximately $7.7 \mathrm{kPa}$. The results for the swirl and axisymmetric injectors are shown as separate curves, as the flow fields are different. These data show a strong trend of Nusselt number increase with frequency for both injection systems. Also shown in Fig. 8 is a linear least-squares fit to the axial injection data (dashed line). It can be seen that the increase with frequency is roughly linear, with the swirl data falling above the line due to the added convection from the swirl. Examination of the linear fit shows that it would intercept the steady turbulent value at a frequency of about $30 \mathrm{~Hz}$, suggesting that the Nusselt number increase with frequency only occurs for frequencies above some minimum value. This is corroborated by both the swirl and axial injection data curves, which if extrapolated would reach the steady turbulent Nusselt number

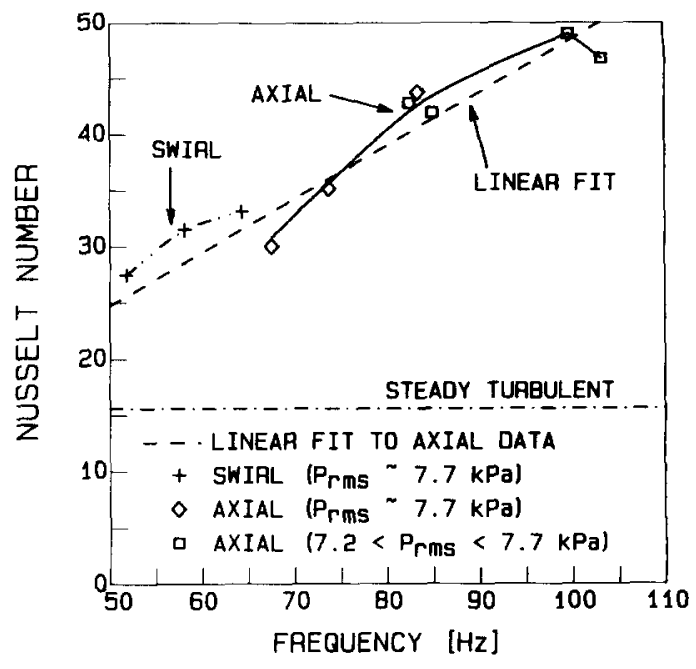

Fig. 8. Time- and space-averaged Nusselt numbers as a function of frequency at a combustion chamber pressure RMS $\left(P_{\mathrm{RMS}}\right)$ of $7.7 \mathrm{kPa}$. Curves are cubic spline fits to data, and dashed line is a linear least-squares fit to the axial injection data.

at frequencies above $30 \mathrm{~Hz}$. This finding is in agreement with the idea that quasi-steady theory, which is independent of frequency, would be expected to hold if the frequency is sufficiently low.

It is also valuable to relate the Nusselt number dependence on frequency with the Nusselt number dependence on $P_{\text {RMs }}$. Because Figs. 6 and 7 show that the slopes of the Nusselt number vs. $P_{\text {RMS }}$ curves increase with frequency, so too the slope of the Nusselt number dependence on frequency must vary with $P_{\text {RMS }}$. Thus, the slope of a Nusselt number vs. frequency plot for a $\boldsymbol{P}_{\mathrm{RMS}}$ less than 7.7 $\mathrm{kPa}$ would have less slope than that shown in Fig. 8 , and at a $P_{\mathrm{RMS}}$ greater than $7.7 \mathrm{kPa}$ the slope would be greater. This simultaneous near-linear dependence on both $P_{\mathrm{RMS}}$ and frequency suggests that, to first order, the Nusselt number enhancement for this pulsing flow is proportional to the product of frequency $(\omega)$ and pulsation amplitude. Because frequency effects occur only above some minimum frequency $\left(\omega_{0}\right)$, this proportionality may be expressed as, $\mathrm{Nu}=f\left(\left(\omega-\omega_{0}\right) *\right.$ pulsation amplitude). As with the increase of Nusselt number with $P_{\text {RMS }}$, Fig. 8 indicates that higher frequencies than those obtained in this study may result in still greater heat transfer enhancements. 


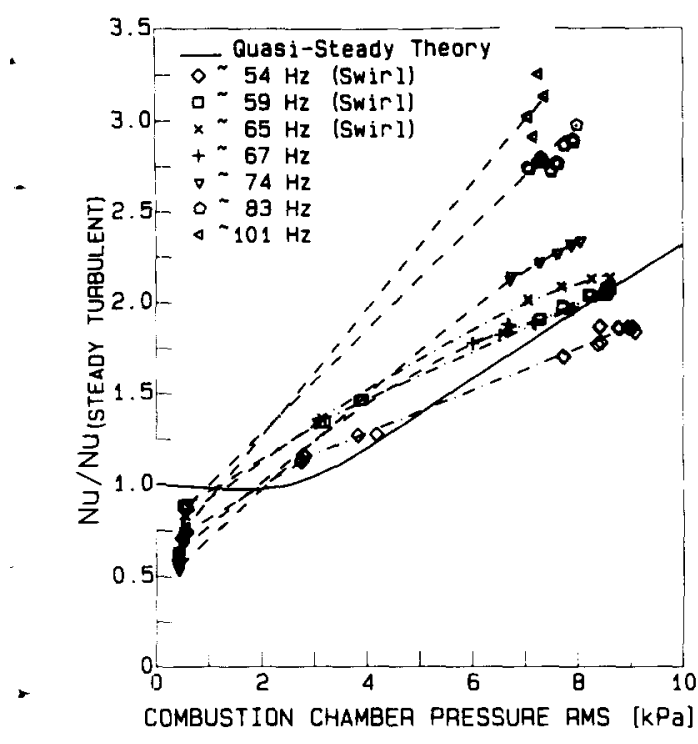

Fig. 9. Comparison of quasi-steady theory with the time- and space-averaged Nusselt numbers from Fig. 6 and 7. The 54$65-\mathrm{Hz}$ cases are for swirl injection and those from $67-101-\mathrm{Hz}$ are axial injection.

Perry and Culick [19] also found convective heat transfer coefficient increases with frequency, although their experimental apparatus was somewhat different than that used here, and their frequencies were much higher.

\section{Comparison with Quasi-Steady Theory}

In Fig. 9 the spatially averaged data from Figs. 6 and 7 are compared with quasi-steady theory (solid line). Since acoustic theory was seen to underpredict the velocity oscillations associated with a given $P_{\mathrm{RMS}}$, and the log-mean calculations overpredict the Nusselt number by $20 \%$, the location of the quasi-steady line may be low relative to the data. Equation 1 shows that quasi-steady theory predicts a Nusselt number increase with oscillation amplitude, but contains no frequency dependence. Therefore, these data, which show a strong Nusselt number dependence on frequency as well as pulsation amplitude, cannot be described by quasisteady theory. The theory does roughly match the data in the $59-67-\mathrm{Hz}$ range. This match with the data at typical pulse combustor frequencies may explain why some previous researchers found agreement with quasi-steady theory (for example, Ref. 4). However, the significant discrepancy of quasi-steady theory from the rest of the data and its lack of frequency information indicate that the success of this correlation is fortuitous. Deviations from quasi-steady theory similar to those shown in Fig. 9 were found by Keil and Baird [16], who studied oscillating flow in a shell-and-tube heat exchanger, albeit at much lower frequencies, 0.4$1.1 \mathrm{~Hz}$.

\section{Mean Flow Rate Effects}

Variation in the mean Reynolds number was obtained by varying the mean mass flow rate of reactants supplied to the combustor, and the results are shown in Fig. 10. Because the magnitude of the energy release also increased with the mean mass flow rate, the data presented in Fig. 10 show an increase in $\boldsymbol{P}_{\mathrm{RMS}}$ for the higher Reynolds number data. The same effect was noted by Keller et al. [8] when the timing of the energy release was optimized. As discussed above, the stability limits of the combustor would not allow it to be

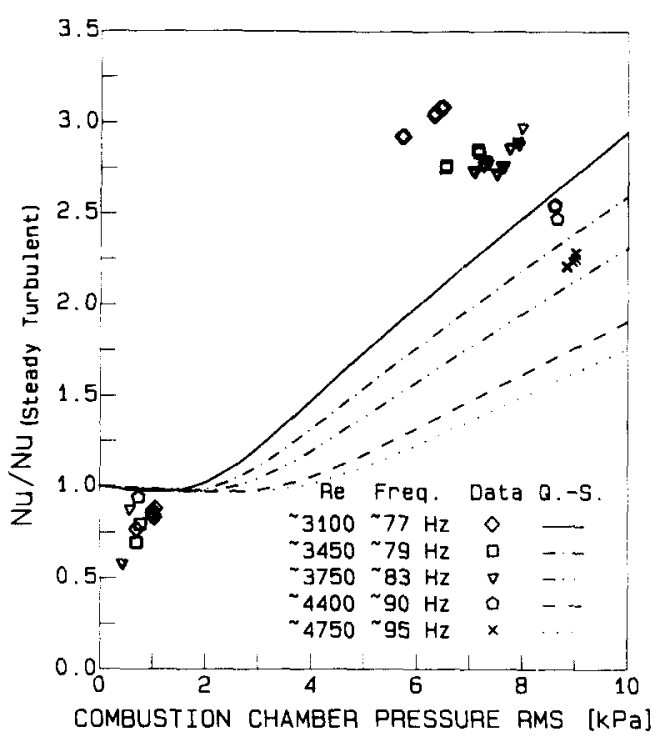

Fig. 10. Mean Reynolds number effect on Nusselt number enhancement relative to steady turbulent flow at the same mean Reynolds number. Properties for each quasi-steady line are evaluated using the mean temperature of the respective experimental data. 
sufficiently detuned to obtain the same $\boldsymbol{P}_{\mathrm{RMS}}$ for each Reynolds number (mass flow rate). Although the geometry was invariant with a constant tail pipe length of $880 \mathrm{~mm}$, the resonant frequency varied with mass flow rate, as noted in Fig. 10, due to temperature and combustion timing effects. In spite of this inability to vary the mean Reynolds number independently of the frequency and pulsation amplitude, trends can be determined from these data.

In Fig. 10 the Nusselt numbers, normalized by their respective steady flow values, predicted from the Colburn correlation, are plotted as a function of $P_{\text {RMS }}$. Again the Nusselt numbers for the nonpulsing cases are less than the expected steady turbulent values. The Nusselt number enhancement for Reynolds numbers 3100,3450 , and 3750 is roughly the same, a factor of about 2.7; however, the frequency and $P_{\mathrm{RMS}}$ to achieve this same enhancement increases with mean Reynolds number. For higher mean Reynolds numbers, 4400 and 4750 , there is a distinct decrease in the enhancement. The general trend of these data is that the heat transfer enhancement remains constant or decreases as the mean Reynolds number increases. This occurs despite accompanying increases in $P_{\mathrm{RMS}}$ and frequency, both of which have been shown (Figs. 6-8) to increase the Nusselt number enhancement. Therefore, if it had been possible to operate the combustor at a constant pulsation frequency and amplitude as the mean Reynolds number was increased, a steady decrease in Nusselt number enhancement would be expected.

Figure 10 also shows a comparison of the variable mean Reynolds number data with quasisteady theory. Because the enhancement predicted by quasi-steady theory varies with mean Reynolds number (see Eq. 1), five curves are shown in the figure. Each quasi-steady curve was calculated using fluid properties based on the appropriate mean temperatures of the respective experimental data. All of the data presented fall above their respective curves. This result may have been anticipated, since the frequencies range from 77 to $95 \mathrm{~Hz}$, all greater than $67 \mathrm{~Hz}$, which was found to fortuitously coincide with quasi-steady theory in Fig. 9. Quasi-steady theory and the experimental data both show a decrease in enhancement with increasing mean Reynolds number and the spread in the data and the theory is similar. This suggests another reason why some previous work was found to correlate with quasi-steady theory and indicates that the ratio of $V_{\text {osc }} / V_{\text {mean }}$ may be an important parameter correlating heat transfer in oscillating flows.

\section{SUMMARY AND CONCLUSIONS}

A heat transfer study in the tail pipe of a pulse combustor was conducted, in which the pulsation frequency, pulsation amplitude, and mean flow rate were systematically varied. The Nusselt numbers in the oscillating flows were found to be, significantly higher than those expected for steady turbulent flow at the same mean Reynolds number. Spatially averaged Nusselt numbers, obtained using the log-mean heat exchanger technique, increased linearly with both pulsation frequency and amplitude, with a maximum measured enhancement of 2.5 times that of steady turbulent flow. The Nusselt number enhancement decreased with increasing mass flow rate despite accompanying increases in oscillation frequency and amplitude. These results indicate that the Nusselt number enhancement for this oscillating flow is a function of the pulsation-amplitude frequency product and the ratio of the oscillating velocity to the mean velocity. Since the pulsation amplitude and $V_{\text {osc }}$ are directly related, and the enhancement effect is only seen for frequencies above some minimum frequency, this may be stated as, $\mathrm{Nu}=$ $f\left(\left(\omega-\omega_{0}\right) * V_{\text {osc }}, V_{\text {osc }} / V_{\text {mean }}\right)$. Independent axially resolved measurements confirmed the large Nusselt number increase with pulsations and demonstrated that entrance effects, although present, were small compared to the Nusselt number enhancement due to resonance.

The data were compared with quasi-steady theory, which is the only available theory in the literature for correlating heat transfer in oscillating flows. Quasi-steady theory correctly predicts increased Nusselt number enhancement with increases in pulsation amplitude, and decreased Nusselt number enhancement with increases in the 
mean Reynolds number. However, quasi-steady theory does not contain frequency information, so it cannot predict the observed Nusselt number increases with frequency. The strong frequency dependence seen in the experimental data show that quasi-steady theory is not adequate for predicting heat transfer in this type of oscillating flow.

The authors would like to express their gratitude to Ichiro Hongo of Toshiba Corporation for his assistance in the data acquisition and to Pamela Barr of Sandia for her preparation of Fig. 2. This work was performed at the Combustion Research Facility at Sandia National Laboratories and was supported by the U.S. Department of Energy, Division of Energy Conversion and Utilization Technologies Program and the Gas Research Institute.

\section{REFERENCES}

1. Putnam, A. A., Belles, F. E., and Kentfield, J. A. C., Prog. Ener. Combust. Sci. 12:43-79 (1986).

2. Zinn, B. T., ASME Paper 84-WA/NCA-19, ASME Winter Annual Meeting, New Orleans, LA, December, 1984.

3. Alhaddad, A. A., and Coulman, G. A., Experimental and Theoretical Study of Heat Transfer in Pulse-Combustion Heaters, Proceedings Vol. I: Symposium on Pulse Combustion Applications, GRI-82/0009.2, Atlanta, GA, March 1982.

4. Hanby, V. 1., ASME J. Eng. Power 91:48-52 (1969).

5. Liao, N. S., Wang, C. C., and Hong, J. T., An Investigation of Heat Transfer in Pulsating Turbulent Pipe Flow, ASME 23rd National Heat Transfer Conference, Denver, Colorado, 1985, Fundamentals of Forced and Mixed Convection, F. A. Kulacki and R. D. Boyd, Eds., HTD, Vol. 42.

6. Galitseyskiy, B. M., and Ryzhov, Yu. A., Heat Transfer Soviet Res., Vol. 9, 9:178-183 (1977).

7. Dec, J. E., and Keller, J. O., Pulse Combustor Tail-Pipe Heat-Transfer Dependence on Frequency, Amplitude, and Mean Flow Rate, ASME Winter Annual Meeting, Chicago, December 1988.

8. Keller, J. O., Dec., J. E., Westbrook, C. K., and Bramlette, T. T.: Combust. Flame, Vol. 75, No. 1, pg. 33, Jan. 1989.

9. Keller, J. O., Westbrook, C. K., Bramlette, T. T., and Dec. J. E., Sandia National Laboratories Report, SAND87-8832.

10. Bramlette, T. T., Sandia National Laboratories Report, SAND87-8622.
11. Dec, J. E., and Keller, J. O., Sandia National Laboratories Report, SAND86-8757.

12. Bradley, D., and Entwistle, A. G., Br. J. Appl. Phys. 12:708-711 (1961).

13. Barnett, D. O., and Vachon, R. I., An Analysis of Convective Heat Transfer for Pulsating Flow in a Tube, Heat Transfer 1970, Vol. 3.

14. Stosic, N., and Hanjalic, K., Numerical Study of Unsteady Convective Heat Transfer in Pulsating Duct Flows, Heat Transfer 1982, Vol. 3.

15. Martinelli, R. C., Boelter, L. M. K., Weinberg, E. B., and Yakahi, S., ASME Trans. 65:798 (1943).

16. Keil, R. H., and Baird, M. H. I., Ind. Eng. Chem. Proc. Des. Dev. 10:473 (1971).

17. Incropera, F. P., and DeWitt, D. P., Fundamentals of Heat Transfer, Wiley, New York, 1981, pp. 411, 507512.

18. Reay, D., J. Inst. Fuel, 22:135-142 (1969).

19. Perry, E. H., and Culick, F. E. C., Combust. Sci. Technol. 9:49-53 (1974).

\section{APPENDIX A}

\section{Acoustic Relationship Between Pressure and Velocity Oscillations}

For a wave in acoustic resonance, the amplitude of the pressure oscillation at the pressure antinode, $P_{\max }$, is related to the amplitude of the velocity oscillation at the velocity antinode, $V_{\text {osc }-\max }$ as,

$$
V_{\text {osc }-\max }=\frac{P_{\max }}{\rho c},
$$

where $\rho$ is the average density and $c$ is the average speed of sound. Since the oscillations are sinusoidal (Fig. 3), $P_{\max }=\sqrt{2} P_{\text {RMS }}$. Equation A1 provides the velocity oscillation amplitude at the tail pipe exit; however the oscillation amplitude decays sinusoidally towards the tail pipe entrance, as seen in Fig. 2. To allow Eq. 1 to be compared with the log-mean data, an average oscillation velocity is required for the velocity ratio $B$. This average velocity oscillation amplitude $V_{\text {osc - avg }}$ was obtained by integrating the sinusoidal velocity distribution from the tail pipe inlet to exit, as shown in Eq. A2.

$$
V_{\text {osc-avg }}=V_{\text {osc }-\max } \int_{\lambda / 4-L}^{\lambda / 4} \sin \left(\frac{2 \pi x}{\lambda}\right) d x,
$$


where $\lambda$ is the acoustic resonance wavelength, determined from the measured combustor frequency and the average speed of sound, $L$ is the length of the tail pipe, and $x$ is distance along the axis of the tail pipe. Performing the integration in Eq. A2 and combining it with Eq. A1 yields

$$
V_{\text {osc }-\mathrm{avg}}=\frac{P_{\max }}{\rho c} \frac{1}{L} \frac{\lambda}{2 \pi} \cos \left(\frac{\pi}{2}-\frac{2 \pi L}{\lambda}\right) .
$$

This value of $V_{\text {osc-avg }}$ was used in the quasi-steady correlation, Eq. 1, to obtain the curves given in Figs. 7 and 8.

\section{APPENDIX B}

\section{Log-Mean Nusselt Number Calculations}

The standard derivation of the log-mean counterflowing heat exchanger formula assumes that the heat exchanger is insulated. Because the outer wall of the tail pipe was uninsulated, the formula was derived accounting for a heat loss from the outer tube to the ambient. This formulation gives the following result for $U$, the overall heat transfer coefficient, which includes both exhaust gas and cooling air convective coefficients and the wall conduction:

$$
U=\frac{\ln \left(\frac{\Delta T_{2}}{\Delta T_{1}}\right)}{\frac{A}{Q}\left(\Delta T_{2}-\Delta T_{1}\right)+\frac{1}{C_{C}}\left(\int_{1}^{2} \frac{d Q_{0} d A}{d Q}-\frac{Q_{0} A}{Q}\right)},
$$

where $\Delta T$ refers to the temperature difference between the exhaust gas and cooling air, with the numbers 1 and 2 referring to the exhaust gas inlet and exit ends of the tail pipe, respectively. $C_{C}$ is the product of the mass flow rate and the constant pressure specific heat of the cooling air. $Q$ is the heat flux from the exhaust gas to the cooling air, $Q_{0}$ is the heat flux from the cooling air to the ambient, and $A$ is the area of the wall of the inner pipe. This equation for $U$ differs from the standard adiabatic formulation only in the addition of the second term in the denominator. This nonadiabatic term is comprised of two terms of opposite sign. Equation B1 may be reduced to its adiabatic form, by assuming the two terms were equal as shown in Eq. B2.

$\int_{1}^{2} \frac{d Q_{0} d A}{d Q}=\frac{Q_{0} A}{Q}$

Although this assumption is not strictly true due to nonlinearities in the heat flux ratio, the integrated effect is expected to be small. Moreover, for typical values in the pulse combustor tail pipe, even if there is no canceling effect of these terms (i.e., $\int_{1}^{2}\left(d Q_{0} d A\right) / d Q=0$ ), the right-hand term in the denominator of Eq. B1 is a factor of 5 smaller than the left-hand term. Hence any errors in dropping the nonadiabatic terms and reducing Eq. B1 to its standard adiabatic form are small.

Received 14 April 1988; revised 21 October 1988 